\title{
A DESCRIPTION OF THE PROJECTIVE STONE ALGEBRAS
}

\author{
by IVO DÜNTSCH
}

(Received 20 August, 1981)

0. Introduction. In his book [6] Grätzer sets a guideline for the research on Stone algebras; in view of his and Chen's triple characterization of Stone algebras [3], he considers a problem for these algebras solved if it can be reduced to a problem for Boolean algebras and one for distributive lattices with 1. In the same book he lists as Problem 53: describe the projective Stone algebras. For a Stone algebra $L$, let $B_{L}$ be its centre and $D_{L}$ its dense set. In order that $L$ be projective, $B_{L}$ has to be a Boolean retract of $B_{F}$ for some free Stone algebra $F$, and $D_{L}$ has to be a retract of $D_{F}$ in the category of distributive lattices with 1 . These conditions are, however, not sufficient, so one hopes to characterize the projective Stone algebras by adding further conditions, and in this spirit we in fact arrive at a description of these algebras. We also show, however, that every such algebra is a retract of some $D_{F}, F$ a free Stone algebra, so we conclude that there is no nice structural characterization of the projective Stone algebras along the line of Grätzer's programme.

The author dedicates this paper to Sylvia Wenderoth; he also would like to thank Sabine Koppelberg for many discussions on the subject, and the referee for pointing out an error in the former version of Lemma 1.3, and supplying the correct version.

1. Preliminaries. A Stone algebra $(L,+, \cdot, *, 0,1)$ is a pseudocomplemented distributive lattice with 0 and 1 , such that $x^{*}+x^{* *}=1$ for all $x \in L$. Throughout the paper any structure will be identified with its underlying set. The set $B_{L}=\left\{x^{*} \mid x \in L\right\}$ is called the centre of $L$; it is the set of complemented elements of $L$, and, as a subalgebra of $L$, it is a Boolean algebra. The set $D_{L}=\left\{x \in L \mid x^{*}=0\right\}$ is called the dense set of $L$, and it is a filter in $L$. Finally, $L$ is a double Stone algebra if every $x \in L$ has a dual pseudocomplement $x^{+}$, and if $x^{+} \cdot x^{++}=0$.

The reader is referred to [6] for the basic facts on Stone algebras. A Stone algebra homomorphism is a lattice homomorphism which also preserves $*, 0$, and 1 .

1.1. Lemma (Chen, Grätzer [3]). Let $L$ and $M$ be Stone algebras, and suppose $f_{1}: B_{L} \rightarrow B_{M}$ is a Boolean homomorphism, and that $f_{2}: D_{L} \rightarrow D_{M}$ is a lattice homomorphism preserving 1. Then there is a Stone algebra homomorphism $f: L \rightarrow M$ such that $f \mid B_{L}=f_{1}$ and $f \mid D_{L}=f_{2}$ if and only if $f_{1}(a) \leqslant f_{2}(x)$ for all $a \in B_{L}$ and $x \in D_{L}$ which satisfy $a \leqslant x$. In this case, the extension $f$ is unique. Furthermore, $f$ is onto (one-to-one) if and only if $f_{1}$ and $f_{2}$ are onto (one-to-one).

If $L$ is a double Stone algebra, this easily implies

1.2. Lemma. Let $L, M, f_{1}$, and $f_{2}$ be defined as in Lemma 1.1., and let $L$ be a double Stone algebra. Then there is an extension $f$ of $f_{1}$ and $f_{2}$ over $L$ if and only if $f_{1}\left(x^{++}\right) \leqslant f_{2}(x)$ for all $x \in D_{L}$.

Note that $f$ does not necessarily preserve ${ }^{+}$.

Glasgow Math. J. 24 (1983) 75-82. 
If $I$ is an ideal of a Stone algebra $L$, then $I$ determines a lattice congruence if we set $x \equiv y$ if there is a $z \in I$ such that $x+z=y+z$, but $L / I$ does not have to be a Stone algebra, and, even when it is, the canonical epimorphism need not preserve *. The following lemma, the proof of which is left to the reader, tells us when it does. Call an ideal $I$ normal if $x \in I$ implies $x^{* *} \in I$; note that a normal ideal has the form $(J]$ for an ideal $J$ of $B_{L}$.

1.3. Lemma. Let $I$ be an ideal of a Stone algebra $L$. Then the induced congruence preserves pseudocomplementation if and only if $I$ is normal.

Note that in particular $L / I$ is a Stone algebra for a Stone algebra $L$ and $I$ a normal ideal of $L$.

Let $\alpha$ be a nonzero cardinal, and for every $i<\alpha$ let $F_{i}$ be the free Stone algebra generated by some element $a_{i 0}$.

1.4. Theorem (Balbes, Horn [1]). Let $F$ be the free product of $\left\{F_{i} \mid i<\alpha\right\}$ in the category of distributive lattices with 0 and 1 . Then $F$ is the free Stone algebra on the free generators $\left\{a_{i 0} \mid i<\alpha\right\}$.

For the rest of the paper $F_{\alpha}$ will be the free Stone algebra on the free generators $\left\{a_{i 0} \mid i<\alpha\right\}$ with $B_{\alpha}$ its centre, and $D_{\alpha}$ its dense set. For every $i<\alpha$ set $a_{i 1}=a_{i 0}^{*}, a_{i 2}=a_{i 0}^{* *}$, and let, for $r \in\{0,1,2\}$,

$$
A^{r}=\left\{a_{i r} \mid i<\alpha\right\} .
$$

Furthermore, set $A=A^{0} \cup A^{1} \cup A^{2}$. Then Theorem 1.4 implies that for every $x \in$ $F_{\alpha} \backslash\{0,1\}$ there are nonempty finite subsets $T_{1}, \ldots, T_{m}$ and $S_{1}, \ldots, S_{r}$ of $A$, such that $x=\sup T_{1} \cdot \ldots \cdot \sup T_{m}$, and $x=\inf S_{1}+\ldots+\inf S_{r}$. The sets used in the representation of an $x \in F_{\alpha}$ are always assumed to be nonempty finite subsets of $A$. If $T$ is such a set, let

$$
\bar{T}=T \cup\left\{a_{i 0} \mid a_{i 2} \in T\right\} .
$$

Then sup $T=\sup \bar{T}$, since $a_{i 0} \leqslant a_{i 2}$. The following two lemmas are taken from Katrinák [7].

1.5. Lemma. Let $T$ be a nonempty finite subset of $A$.

(1) $\sup T=1$ if and only if there is an $i<\alpha$, such that $a_{i 1}, a_{i 2} \in T$. [1])

(2) inf $T=0$ if and only if there is an $i<\alpha$, such that $a_{i 0}, a_{i 1} \in \bar{T}$. (cf. also Balbes, Horn

(3) $\sup T \in D_{\alpha}$ if and only if there is an $i<\alpha$, such that $a_{i 0}, a_{i 1} \in \bar{T}$.

1.6. Lemma. Let $x, y \in F_{\alpha}, x=\sup T_{1} \cdot \ldots \cdot \sup T_{m}, y=\sup W_{1} \ldots . \sup W_{p}$, such that sup $W_{j} \neq 1$ for all $j \leqslant p$. Then $x \leqslant y$ if and only if for every $j \leqslant p$ one of the following conditions holds:

(1) there is an $i \leqslant m$, such that $T_{i}$ is a subset of $\bar{W}_{j}$;

(2) for all $i \leqslant m, T_{i}$ is not a subset of $\bar{W}_{j}$, and

$$
\sup \left(T_{1} \backslash \bar{W}_{j}\right) \cdot \ldots \cdot \sup \left(T_{m} \backslash \bar{W}_{j}\right)=0 .
$$


In the same paper Katrinák has also shown that $B_{\alpha}$ is the free Boolean algebra freely generated by $\left\{a_{i 2} \mid i<\alpha\right\}$.

2. The characterization. Recall that a Stone algebra $L$ is projective if and only if it is a retract of some $F_{\alpha}$. It follows from 1.1 that then $B_{L}$ is a Boolean retract of $B_{\alpha}$, i.e. a projective Boolean algebra by Katrinák's result, and that $D_{L}$ is a retract of $D_{\alpha}$ in the category of distributive lattices with 1 . We have shown in [5]

\subsection{Lemma. A projective Stone algebra is a double Stone algebra.}

Proof. $F_{1}$ is isomorphic to its dual lattice, so it follows from 1.4 that any free Stone algebra is a double Stone algebra. Now let $L$ be projective, and $f: F_{\alpha} \rightarrow L$ be an onto Stone homomorphism. Then there is a Stone homomorphism $g: L \rightarrow F_{\alpha}$, such that $f \circ g=$ id $L$. For $x \in L$ define $x^{+}=f\left(g(x)^{+}\right)$; it is not hard to show that $x^{+}$is the dual pseudocomplement of $x$, and that $x^{+} \cdot x^{++}=0$. $f(y)^{+}$.

Note that neither $f$ nor $g$ necessarily preserves ${ }^{+}$, but that for any $y \in g[L], f\left(y^{+}\right)=$

In order to arrive at our description of the projective Stone algebras, we shall first exhibit a certain class of such algebras. As a preparation we shall need one more lemma, the proof of which is an easy consequence of 1.6 and is left to the reader.

2.2. Lemma. Suppose $b, c \in B_{\alpha}$, and $M_{1}$ and $M_{2}$ are nonempty finite subsets of $A^{0}$, which are disjoint. Then $\inf M_{1} \cdot b \leqslant \sup M_{2}+c$ implies $\inf M_{1} \cdot b \leqslant c$.

Note that, by the lemma, $a_{i 0} \neq c$ and $j \neq i$ imply $a_{i 0} \neq a_{j 0}+c$.

2.3. THEOREM. Let I be a normal ideal of $F_{\alpha}$, such that $L=F_{\alpha} / I$ has a projective centre. Then $L$ is a projective Stone algebra.

Proof. Let $f: F_{\alpha} \rightarrow L$ be the canonical epimorphism, and let $g_{1}: B_{L} \rightarrow B_{\alpha}$ be a Boolean homomorphism such that $f \circ g_{1}=$ id $B_{L}$. Let

$$
J=\left\{i<\alpha \mid f\left(a_{i k}\right) \notin\{0,1\} \text { for some } k \in\{0,1,2\}\right\} ;
$$

we can assume without loss of generality that $J$ is nonempty. Then,

$$
S=\left\{f\left(a_{i k}\right) \mid i \in J, k=0,1,2\right\}
$$

is a set of generators for $L$. Now we define a mapping $r: S \rightarrow F_{\alpha}$ by

$$
r\left(f\left(a_{i k}\right)\right)=\left\{\begin{array}{lll}
g_{1}\left(f\left(a_{i k}\right)\right) & \text { if } k=1,2, \\
g_{1}\left(f\left(a_{i 2}\right)\right) \cdot\left(a_{i 0}+a_{i 1}\right) & \text { if } \quad k=0 .
\end{array}\right.
$$

For all $i \in J, a_{i 0}$ is not an element of $I$, hence $a_{i 0} \neq b$ for all $b \in I \cap B_{\alpha}$. It is then implied by 2.2 that for all $i, j \in J, i \neq j$, we have $f\left(a_{i 0}\right) \neq f\left(a_{j 0}\right)$; hence, $r$ is well-defined.

Suppose

$$
T=\left\{f\left(a_{i_{0} 0}\right), \ldots, f\left(a_{i_{k}}\right), f\left(b_{1}\right), \ldots, f\left(b_{r}\right)\right\}
$$


and

$$
W=\left\{f\left(a_{j_{1} 0}\right), \ldots, f\left(a_{j_{s} 0}\right), f\left(c_{1}\right), \ldots, f\left(c_{\imath}\right)\right\}
$$

are subsets of $S$, where $b_{1}, \ldots, b_{r}$ and $c_{1}, \ldots, c_{t}$ are elements of $A^{1} \cup A^{2}$. Let inf $T \leqslant$ sup $W$; it is well-known that $r$ can be extended to a lattice homomorphism over $L$ if inf $r[T] \leqslant \sup r[W]$; the extension preserves 0 (1), if inf $T=0$ (sup $T=1$ ) implies inf $r[T]=0(\sup r[T]=1)$.

inf $T \leqslant \sup W$ implies that there is an $x \in I$, such that

$$
a_{i_{1} 0} \cdot \ldots \cdot a_{i_{k} 0} \cdot b_{1} \cdot \ldots \cdot b_{r} \leqslant a_{i_{1} 0}+\ldots+a_{j, 0}+c_{1}+\ldots+c_{t}+x .
$$

Since $I$ is normal, we can suppose without loss of generality that $x$ is an element of $B_{\alpha}$. If $a_{i_{n} 0}=a_{j_{m} 0}$ for some $n \leqslant k, m \leqslant s$, then obviously inf $r[T] \leqslant \sup r[W]$, so suppose without loss of generality that $\left\{a_{i_{1} 0}, \ldots, a_{i_{k} 0}\right\}$ and $\left\{a_{j_{1} 0}, \ldots, a_{j_{0} 0}\right\}$ are disjoint. Lemma 2.2 tells us now that

$$
a_{i_{1} 0} \cdot \ldots \cdot a_{i_{k} 0} \cdot b_{1} \cdot \ldots \cdot b_{r} \leqslant c_{1}+\ldots+c_{t}+x .
$$

Since the right side of the inequality is Boolean, this in turn implies

hence

$$
a_{i_{1} 2} \cdot \ldots \cdot a_{i_{k} 2} \cdot b_{1} \cdot \ldots \cdot b_{r} \leqslant c_{1}+\ldots+c_{t}+x
$$

$$
\left.g_{1}\left(f\left(a_{i_{1} 2}\right)\right) \cdot \ldots \cdot g_{1} f\left(a_{i_{k} 2}\right)\right) \cdot g_{1}\left(f\left(b_{1}\right)\right) \cdot \ldots \cdot g_{1}\left(f\left(b_{r}\right)\right) \leqslant g_{1}\left(f\left(c_{1}\right)\right)+\ldots+g_{1}\left(f\left(c_{t}\right)\right),
$$

and therefore

$$
\begin{aligned}
g_{1}\left(f\left(a_{i_{1} 2}\right)\right) \cdot\left(a_{i_{1} 0}+a_{i_{1} 1}\right) \cdot \ldots \cdot g_{1}\left(f\left(a_{i_{2}}\right)\right) \cdot\left(a_{i_{k} 0}+a_{i_{k} 1}\right) \cdot g_{1}\left(f\left(b_{1}\right)\right) & \ldots \cdot g_{1}\left(f\left(b_{r}\right)\right) \\
& \leqslant g_{1}\left(f\left(c_{1}\right)\right)+\ldots+g_{1}\left(f\left(c_{t}\right)\right)
\end{aligned}
$$

This easily implies inf $r[T] \leqslant \sup r[W]$. It is also not hard to show that inf $T=$ $0(\sup T=1)$ implies inf $r[T]=0(\sup r[T]=1)$, so $r$ can be extended to a lattice homomorphism $\bar{r}: L \rightarrow F_{\alpha}$, which preserves 0 and 1 .

To show that $\bar{r}$ preserves pseudocomplementation, it suffices to show that $\bar{r}\left(f\left(a_{i 0}+\right.\right.$ $\left.\left.a_{i 1}\right)\right)$ is in $D_{\alpha}$ :

$$
\begin{aligned}
\bar{r}\left(f\left(a_{i 0}+a_{i 1}\right)\right)^{*} & =\left(r\left(f\left(a_{i 0}\right)\right)+r\left(f\left(a_{i 1}\right)\right)\right)^{*} \\
& =r\left(f\left(a_{i 0}\right)\right)^{*} \cdot r\left(f\left(a_{i 1}\right)\right)^{*} \\
& =\left(g_{1}\left(f\left(a_{i 2}\right)\right) \cdot\left(a_{i 0}+a_{i 1}\right)\right)^{*} \cdot g_{1}\left(f\left(a_{i 1}\right)\right)^{*} \\
& =g_{1}\left(f\left(a_{i 2}\right)\right)^{*} \cdot g_{1}\left(f\left(a_{i 1}\right)\right)^{*} \\
& =0 .
\end{aligned}
$$

Furthermore,

$$
\begin{aligned}
f\left(r\left(f\left(a_{i 0}\right)\right)\right) & =f\left(g_{1}\left(f\left(a_{i 2}\right)\right) \cdot\left(a_{i 0}+a_{i 1}\right)\right) \\
& =f\left(a_{i 2}\right) \cdot\left(f\left(a_{i 0}\right)+f\left(a_{i 1}\right)\right) \\
& =f\left(a_{i 0}\right) .
\end{aligned}
$$

Thus, $L$ is a retract of $F_{\alpha}$, and therefore projective. 
For those readers who are familiar with the topological duality of Stone algebras (see Priestley [8]), the following facts seem worthy of mention. If $M$ is an arbitrary Stone algebra, the topological and order components of $S(M)$, the Stone space of $M$, are of the form $\{Q \in S(M) \mid P \subseteq Q\}$, where $P$ is a minimal prime ideal of $M$ (cf. [5]). The prime ideals of $L$ are in one-to-one correspondence with those prime ideals of $F_{\alpha}$ which contain $I$, and $I$ is the intersection of a family of minimal prime ideals of $F_{\alpha}$. Thus, $S(L)$ consists of "whole" components of $S\left(F_{\alpha}\right)$.

The proof of the following corollary can be derived mutatis mutandis from the proof of Theorem 3.4 of [5], of which it is a generalization.

2.4. Corollary. Let $L$ be a projective Stone algebra and let I be a normal ideal of $L$. If $L / I$ has a projective centre, then it is a projective Stone algebra.

The fact that every countable Boolean algebra is projective provides us with

2.5. Corollary. If $L$ is a projective Stone algebra with a countable centre and I a normal ideal of $L$, then $L / I$ is projective.

Now we can describe the projective Stone algebras.

2.6. Theorem. A Stone algebra $L$ is projective if and only if it is a double Stone algebra and there are a free Stone algebra $F$ and an onto Stone homomorphism $f: F \rightarrow L$, such that the following two conditions hold.

(1) There is a Boolean homomorphism $g_{1}: B_{L} \rightarrow B_{F}$ such that $f \circ g_{1}=$ id $B_{L}$.

(2) There is a lattice homomorphism $g_{2}: D_{L} \rightarrow D_{F}$ such that $f \circ g_{2}=\mathrm{id} D_{L}, g_{2}(1)=1$, and $f\left(g_{2}(x)^{+}\right)=x^{+}$for all $x \in D_{L}$.

Proof. Because of 2.1 and the remarks at the beginning of this section we need only show sufficiency.

Suppose $I$ is the ideal of $F$ which is generated by $f^{-1}(0) \cap B_{F}$. Then $I$ is normal, and the centre of $F / I$ is isomorphic to the centre of $L$ which is projective by our hypothesis. Hence, $F / I$ is a projective Stone algebra by Theorem 2.3. Let $s: F \rightarrow F / I$ be the canonical epimorphism; note that $s$ also preserves dual pseudocomplementation. We shall show now that $L$ is a retract of $F / I$.

Define $q: F / I \rightarrow L$ by $q(s(x))=f(x)$ for all $x \in F$. Suppose $s(x)=s(y)$; then there is an $a \in I \cap B_{F}$, such that $x+a=y+a$. Hence, $f(x)=f(y)$, and $q$ is well defined. Obviously, $q$ is onto, and $q_{1}$, the restriction of $q$ to the centre of $F / I$, is an isomorphism. Let $g_{1}: B_{L} \rightarrow B_{F}$ be a Boolean homomorphism which satisfies $f \circ g_{1}=$ id $B_{L}$, and set $p_{1}=s \circ g_{1}$. Then $p_{1}=q_{1}^{-1}$.

Let $g_{2}: D_{L} \rightarrow D_{F}$ be a mapping having the properties prescribed in (2) above. Note that $f\left(g_{2}(x)^{+}\right)=x^{+}$implies that $f\left(g_{2}(x)^{++}\right)=x^{++}$, since $g_{2}(x)^{++}=g_{2}(x)^{+*}$, and $f$ preserves $*$. Let $p_{2}=s \circ g_{2}$. Then $p_{2}$ is a lattice homomorphism from $D_{L}$ into the dense set of $F / I$, which preserves 1 . Furthermore, for all $x \in D_{F}$,

$$
\begin{aligned}
q\left(p_{2}(f(x))\right) & =q\left(s\left(g_{2}(f(x))\right)\right) \\
& =f\left(g_{2}(f(x))\right) \\
& =f(x),
\end{aligned}
$$


so all that is left to show is that $p_{1}, p_{2}$ can be extended over $L$. So, let $y$ be an element of $D_{L}$. Then

$$
\begin{aligned}
q\left(p_{2}(y)^{++}\right) & =q\left(s\left(g_{2}(y)\right)^{++}\right) \\
& =q\left(s\left(g_{2}(y)^{++}\right)\right), \quad \text { since } s \text { preserves }{ }^{+}, \\
& =f\left(g_{2}(y)^{++}\right) \\
& =f\left(g_{2}(y)\right)^{++}, \quad \text { by our hypothesis, } \\
& =y^{++} .
\end{aligned}
$$

Since $p_{1}=q_{1}^{-1}$, we have $p_{1}\left(y^{++}\right)=p_{2}(y)^{++} \leqslant p_{2}(y)$, so there is an extension of $p_{1}$ and $p_{2}$ over $L$ by 1.2 , and thus $L$ is projective.

\section{The circle game}

3.1. Theorem. Let $\alpha$ be infinite. Then $F_{\alpha}$ is a retract of $D_{\alpha}$ in the category of distributive lattices with 1 .

Proof. Let $L$ be the sublattice of $F_{\alpha}$ which is generated by

$$
\left\{a_{i k} \mid 0<i<\alpha, k=0,1,2\right\},
$$

and let

$$
M=\left\{a_{00}+a_{01}+x \mid x \in L\right\} .
$$

Then $L$ and $M$ are isomorphic to $F_{\alpha}$, and $M$ is a 1 -sublattice of $D_{\alpha}$. Define $f: D_{\alpha} \rightarrow M$ by

$$
f(y)=a_{00}+a_{01}+y .
$$

If $f$ is well-defined, it obviously is a retraction, so suppose $y$ is an element of $D_{\alpha}$. We have to show that $f(y)$ is an element of $M$, i.e. we are looking for an element $x$ of $L$ which satisfies

$$
a_{00}+a_{01}+y=a_{00}+a_{01}+x .
$$

Since $L$ is a lattice, and $f$ is a lattice homomorphism, we can suppose $y=\sup T$ for some nonempty finite subset of $A$ (cf. 1.4). If $a_{02} \leqslant y$, then $f(y)=1$, and if $y=a_{00}+a_{01}$, then $f(y) \in M$. So, suppose $a_{02} \notin T$, and $T \neq\left\{a_{00}, a_{01}\right\}$. Now set $W=T \backslash\left\{a_{00}, a_{01}\right\}$; then

$$
W \cup\left\{a_{00}, a_{01}\right\}=T \cup\left\{a_{00}, a_{01}\right\},
$$

and $\sup W$ is an element of $L$. We then have

$$
\begin{aligned}
a_{00}+a_{01}+y & =a_{00}+a_{01}+\sup T \\
& =\sup \left(T \cup\left\{a_{00}, a_{01}\right\}\right) \\
& =\sup \left(W \cup\left\{a_{00}, a_{01}\right\}\right) \\
& =a_{00}+a_{01}+\sup W .
\end{aligned}
$$


Hence, $M$-and therefore $F_{\alpha}$-is a retract of $D_{\alpha}$ in the category of distributive lattices with 1.

Incidentally, we have shown that $\left[a_{i 0}+a_{i 1}\right)$ is isomorphic to $F_{\alpha}$ for any $i<\alpha$.

3.2. COROLlary. If $L$ is a projective Stone algebra such that $|L| \leqslant \alpha$, then $L$ is a retract of $D_{\alpha}$ in the category of distributive lattices with 1 .

A structural characterization of the projective Stone algebras in the sense of Grätzer's programme has to make use of the retracts of $D_{\alpha}$, but we have just seen that among those retracts are, for infinite $\alpha$, all the projective Stone algebras with cardinality less than or equal to $\alpha$. Thus, we have come full circle.

4. Odds and ends. Let us take a brief look at the free objects in the category of double Stone algebras (and double Stone homomorphisms). It was observed in [4] by Davey and Goldberg that, by analogy with Theorem 1.4, the free double Stone algebra on $\alpha$ free generators is the coproduct in the category of distributive lattices with 0 and 1 of $\alpha$ copies of the free double Stone algebra $F$ on one generator. The ordered set of prime ideals of $F$ looks like this:

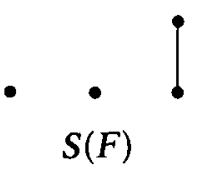

It follows, e.g. from Theorem 3.9 of [5], that $F$ is projective as a Stone algebra. Since the coproduct of projective Stone algebras is projective, and every morphism of double Stone algebras is a morphism of Stone algebras, we arrive at

\subsection{TheOREM. Every projective double Stone algebra is projective as a Stone algebra.}

It is worth noticing that by results of Priestley [8], a free double Stone algebra cannot be the image of a free Stone algebra by a double Stone homomorphism, so indeed Theorem 4.1 makes sense. On the other hand, one can easily show that the free Stone algebra on one generator is projective in the category of double Stone algebras. Hence, every free Stone algebra has this property. The following example shows that this cannot be generalized to arbitrary projective Stone algebras: Let $P$ be a minimal prime ideal of a free Stone algebra $F$, such that $F / P$ is not the two-element Boolean algebra. Then $F / P$ is a projective Stone algebra, whose dense set and dually dense set have a nonempty intersection. This implies that $F / P$ cannot be embedded in a free double Stone algebra by a double Stone homomorphism.

\section{REFERENCES}

1. R. Balbes and A. Horn, Stone lattices, Duke Math. J. 37 (1970), 537-545.

2. R. Balbes and G. Grätzer, Injective and projective Stone algebras, Duke Math. J. 38 (1971), 339-347.

3. C. C. Chen and G. Grätzer, Stone lattices I, Canad. J. Math. 21 (1969), 884-894. 
4. B. Davey and M. Goldberg, The free $p$-algebra generated by a distributive lattice, Algebra Universalis 11 (1980), 90-100.

5. I. Düntsch, Projectivity, prime ideals, and chain conditions of Stone algebras, Algebra Universalis 14 (1982), 167-180.

6. G. Grätzer, Lattice theory (Freeman, 1971).

7. T. Katrinák, Die freien Stoneschen Verbände und ihre Tripelcharakterisierung, Acta Math. Acad. Sci. Hungar. 23 (1972), 315-326.

8. H. A. Priestley, Stone lattices: A topological approach, Fund. Math. 84 (1974), 127-143.

Freie Universität BerLIN

FB 10

GARYSTR. 21

1000 BERLIN 33

W. Germany
Present address: Department of Mathematics BAYERO UNIVERSITY P.M.B. 3011 KANO, NigERIA 